Questions vives

\section{Questions Vives}

Recherches en éducation

Vol.8 $n^{\circ} 15 \mid 2011$

Égalité filles-garçons : où en est-on?

\title{
Qu'est-ce qui fait courir les filles vers la classe préparatoire scientifique Biologie, Chimie, Physique et Sciences de la Terre (BCPST) ?
}

What attracts girls in science classes: Biology, Chemistry, Physics and Earth Studies?

\section{Christine Fontanini}

\section{(2) OpenEdition}

Journals

Édition électronique

URL : http://journals.openedition.org/questionsvives/805

DOI : 10.4000 /questionsvives.805

ISBN : 978-2-8218-1090-7

ISSN : $1775-433 \mathrm{X}$

Éditeur

Université Aix-Marseille (AMU)

Édition imprimée

Date de publication : 15 septembre 2011

ISBN : 978-2-912643-39-1

ISSN : $1635-4079$

Référence électronique

Christine Fontanini, «Qu'est-ce qui fait courir les filles vers la classe préparatoire scientifique Biologie, Chimie, Physique et Sciences de la Terre (BCPST) ? », Questions Vives [En ligne], Vol. 8 n¹5 | 2011, mis en ligne le 10 octobre 2011, consulté le 19 avril 2019. URL : http://journals.openedition.org/ questionsvives/805; DOI : 10.4000/questionsvives.805

Ce document a été généré automatiquement le 19 avril 2019.

\section{cc)}

Questions Vives est mis à disposition selon les termes de la licence Creative Commons Attribution -

Pas d'Utilisation Commerciale - Pas de Modification 4.0 International. 


\title{
Qu'est-ce qui fait courir les filles vers la classe préparatoire scientifique Biologie, Chimie, Physique et Sciences de la Terre (BCPST)?
}

\author{
What attracts girls in science classes: Biology, Chemistry, Physics and Earth \\ Studies?
}

\section{Christine Fontanini}

\section{Introduction}

1 En France, à la rentrée 2007, 9,5\% des bacheliers généraux ou technologiques ont intégré une Classe Préparatoire aux Grandes Ecoles (CPGE) en première année. Ils/elles sont principalement issu-e-s des filières générales de l'enseignement secondaire $(95,6 \%)$ et peu des filières technologiques $(4,4 \%$ ) (Pons, 2007). Les filles représentent $42,7 \%$ du total des élèves en CPGE. Elles sont majoritaires en classes préparatoires littéraires $(74,1 \%)$, à parité en CPGE économiques et commerciales $(54,8 \%)$ et minoritaires en CPGE scientifiques $(30,5 \%)$ (Repères et références statistiques sur les enseignements, la formation et la recherche, 2010). Toutefois, leur part selon les classes scientifiques est contrastée selon les filières : $70 \%$ en BCPST (Biologie, Chimie, Physique, Sciences de la Vie et de la Terre) ; $27,9 \%$ en maths/physique/sciences de l'ingénieur et $10,8 \%$ en physiquetechnologie (Pons, 2007).

2 Notre recherche vise à comprendre pourquoi les filles sont aussi nombreuses à s'engager vers la préparation BCPST alors qu'elles restent minoritaires dans les deux autres spécialités scientifiques (Pons, 2007). Comment se sont construits pour ces étudiants et étudiantes, leur projet et leur parcours scolaire vers cette classe préparatoire «biovéto »? Quelles sont leurs motivations pour cette filière menant aux professions de 
vétérinaire et d'ingénieur agronome? Quelles sont les écoles les plus convoitées? Quels sont leurs projets professionnels? Sont-ils différents entre les filles et les garçons?

\section{Présentation de la recherche : problématique, hypothèse et méthodologie}

\section{Problématique et hypothèse}

3 A notre connaissance, peu de recherches ont été menées sur les classes préparatoires en général (Belhoste, 2003 ; Bourdieu, 1989 ; Delavault, 1998 ; Fontanini, 1999 ; Adangnikou \& Paul, 2004 ; Dutercq, 2008) et encore moins précisément sur les BCPST. Quelques études portant sur les étudiant-e-s préparant des concours, notamment prestigieux, se penchent plutôt sur leurs difficultés psychologiques face au travail et à la pression subie (Huerre, 2005 ; François-Poncet \& Braconnier, 1998). Ces dernières années, plusieurs rapports officiels se sont particulièrement intéressés à la diversification des classes préparatoires et des grandes écoles, le plus récent datant de 2010 (Dardelet, 2010). Par ailleurs, BuissonFenet et Landrier (2008) ont mené une recherche sur la discrimination positive avec le cas d'une classe préparatoire en ZEP.

4 Toutefois, plusieurs recherches ont montré que le prélude à l'orientation des filles et des garçons dans l'enseignement supérieur correspond, hormis les résultats scolaires, en partie à la socialisation des élèves et à leur choix d'orientation dans le secondaire. Filles et garçons ne sont pas encore éduqués de la même manière dans les familles (Duru Bellat, 1990; Gautier, 2008). A l'école, filles et garçons sont confrontés à une construction scolaire des différences entre les sexes. Les interactions en classe entre enseignant-e-s et enseigné-e-s, l'évaluation et le contenu des programmes sont en général favorables aux garçons engendrant une moindre confiance en soi des filles et des attitudes différentes entre les deux sexes face aux divers domaines du savoir (Duru Bellat, 1994, 1995).

5 Selon Jacques (2003, p. 70), les « filles minorent plus fréquemment que les garçons l'autoévaluation de leur potentiel scolaire dont trois indicateurs ont été mis en évidence: l'estimation des chances de réussir le baccalauréat, l'engagement dans des sections prestigieuses, les intentions post-baccalauréat. Les facteurs de cette pondération minorante sont le niveau socioculturel familial et la valeur scolaire objective ».

6 Les orientations différenciées selon le sexe sont aussi à mettre en relation avec les représentations que les adolescent-e-s se font des professions (Mosconi \& Stevanovic, 2007). Ces dernières peuvent être considérées comme "féminines ou masculines » en sachant que cela ne "concerne pas seulement le fait qu'elles soient majoritairement investies par l'un ou l'autre sexe, mais également qu'elles conviennent mieux aux un-e-s ou aux autres sur des critères d'aptitudes, d'intérêts, de personnalité, de caractéristiques physiques requises, de conditions de travail...» (Vouillot, 2007, p. 94). Ainsi, les filles sont généralement plus tentées par (et dirigées vers) les métiers relationnels et de soin/prise en charge d'autrui comme secrétaire, psychologue, professeur, infirmière... Les garçons sont davantage attirés par (et orientés vers) des métiers scientifiques et techniques.

7 Une autre explication a été proposée par Duru Bellat (1990) qui avance que les filles font des choix réalistes de compromis dans leurs orientations en anticipant un besoin de temps libre. Ainsi, elles choisissent des carrières moins prestigieuses et moins prenantes qui leur permettront de travailler à temps partiel ou avec des horaires de travail souples. 
8 Enfin, une dernière interprétation consiste en l'idée d'une plus grande polyvalence des filles dans leur réussite scolaire et d'une plus grande liberté dans leurs choix d'études (Ferrand, Imbert \& Marry, 1996). Veleine (2004, p. 30) rejoint cette explication : « Elles ont le sentiment d'avoir l'autorisation de choisir leur voie, de changer de cap si nécessaire (mais aussi des pauses éventuellement dans leur vie professionnelle) ».Ainsi, les filles auraient plus la possibilité de choisir une filière d'ingénieur agronome en raison de leur plus grand attrait pour la biologie que les garçons.

9 D'ailleurs, les filles choisissent plus que les garçons, en terminale $S$ (dominante Sciences de la Vie et de la Terre), l'enseignement de spécialité les Sciences de la Vie et de la Terre ( $57,5 \%$ de filles) que la physique-chimie ( $46,9 \%$ de filles), les mathématiques ( $38,5 \%$ de filles) et les sciences de l'ingénieur (13,3\% de filles) (Repères et références statistiques, 2010), ce qui réduit considérablement leurs possibilités d'intégrer certaines filières à dominante maths-physique comme les classes préparatoires. Ainsi, elles se tournent plus vers des études de biologie (en classes préparatoires ou à l'université) ou de médecine.

10 Ces différents éléments nous amènent à poser comme hypothèse que les filles sont largement majoritaires en classes préparatoires BCPST car filles et garçons ne s'orientent pas dans cette préparation pour les mêmes motivations.

11 Notre recherche s'inscrit dans le cadre des travaux portant sur l'orientation des élèves (Guichard, 1993, 2006 ; Bourdieu, 1970, 1981 ; Boudon, 1973 ; Baudelot \& Establet, 1992 ; Boutinet, 2001) et aussi plus spécifiquement sur l'orientation des filles dans les filières scientifiques au niveau secondaire et supérieur (Duru Bellat, 1990; Mosconi, 1994; Fontanini, 1999 ; Stevanovic, 2006). Nous faisons également référence aux représentations des métiers chez les adolescent-e-s (Mosconi \& Stevanovic, 2007).

\section{Méthodologie}

12 Pour tester notre hypothèse, nous avons mené une recherche par questionnaire, courant septembre 2008, auprès de 163 élèves de 4 classes préparatoires BCPST de première année (113 filles et 50 garçons) des 3 lycées toulousains proposant cette filière dans l'académie. Deux d'entre eux sont situés en centre ville et dépendent du ministère de l'Education nationale ; le troisième se trouve en banlieue sud-est très proche et relève du ministère de l'agriculture et de la pêche. La composition de notre échantillon selon le sexe correspond à celle des classes préparatoires BCPST au niveau national (70 \% selon Pons, 2007). La passation des questionnaires s'est faite au moment d'un cours d'un-e professeur-e. Nous étions présente dans la salle ce qui leur permettait de nous demander éventuellement des précisions sur les questions posées. En trente minutes, le questionnaire était en général rempli. Nous avons opté pour la méthode du questionnaire car elle nous semblait appropriée pour recueillir quantitativement des données factuelles sur ces préparationnaires mais aussi leurs motivations et aspirations. Ce mode de recueil convient bien à cette population qui a peu de temps libre.

13 Enfin, cette méthode a permis que tous les élèves d'une même classe remplissent leur questionnaire au même moment ce qui signifie qu'ils/elles étaient tous et toutes dans les mêmes conditions (locaux, horaires) et que nous pouvions les récupérer immédiatement. Nous avons donc eu un taux de retour de $100 \%(n=163)$. Les données ont été saisies et traitées avec le logiciel Sphinx et nous avons reçu l'appui logistique et financier de la Mission égalité filles-garçons du Rectorat de Midi-Pyrénées pour notre recherche. 


\section{Brève présentation de la classe préparatoire scientifique BCPST}

14 Très peu d'études spécifiques ont été conduites sur les classes préparatoires BCPST, à l'exception de celles menées par le Ministère de l'Education Nationale, de l'Enseignement Supérieur et de la Recherche via les rectorats qui nous donnent des données statistiques, et le Service des Concours Agronomiques et Vétérinaires qui fournit un rapport annuel sur les résultats aux concours de la filière BCPST. Une étude exploratoire sur ces classes a été publiée en 2001 et une étude qualitative auprès des classes préparatoires BCPST l'a été en janvier 2002 par un cabinet de consultants suite à une commande demande du Ministère de l'Agriculture, via sa Direction Générale de l'Enseignement et de la Recherche.

15 Jusqu'en 2003, il existait une classe préparatoire scientifique BCPST qui menait aux concours des écoles d'agronomie et une préparation spécifique au concours commun des quatre écoles vétérinaires. Cette dernière ne durait qu'un an (sauf redoublement) au lieu de deux pour les autres préparations. Cette classe préparatoire scientifique "vétérinaire " ne menait qu'à un seul concours très sélectif puisque par exemple, en 2001-2002, sur 1257 élèves inscrits en préparation, seulement 400 trouvaient une place dans une des quatre écoles, soit une proportion de $30 \%$ environ (Pauly \& Mandry, 2003). En outre, on ne pouvait se présenter qu'à deux reprises. En cas d'échec définitif au concours, les étudiant-e-s n'avaient guère de possibilités de réorientation. C'est pourquoi, en 2003, cette classe préparatoire est passée à deux années d'études, comme toutes les autres, et a fusionné avec celle qui préparait aux concours des écoles d'agronomie.

Désormais, après cette préparation BCPST, les étudiant-e-s peuvent passer le concours commun vétérinaire et ceux des écoles d'agronomie (ENSA), des écoles nationales d'ingénieurs des travaux agricoles (ENITA). Les concours A des écoles d'agronomie et vétérinaires ont permis en 2005 à 1165 candidat-e-s sur 2397 inscrit-e-s d'intégrer 34 écoles ou spécialités d'école qui recrutent sur cette banque de concours, soit 48,6\% des inscrit-e-s. Si l'on tient compte des candidat-e-s qui ont intégré les autres écoles accessibles par les deux autres banques de la filière BCPST, le pourcentage global d'intégré-e-s par rapport au nombre d'inscrit-e-s s'établit à 55,8 \% (Rapport sur les concours A, filière BCPST, session 2005).

17 Le gros du vivier des écoles vétérinaires provient des classes préparatoires BCPST puisque $86 \%$ des places sont réservées à ces élèves. Le concours des quatre écoles vétérinaires de France reste très sélectif, après une préparation $\mathrm{BCPST}$, puisque le taux de réussite est de 13 \% en moyenne ces dernières années (Rapport sur les concours A, filière BCPST, session 2005). On ne peut le passer que deux fois dans sa vie avec une limite d'âge pour une première inscription à 22 ans. Finalement, avec la nouvelle classe préparatoire BCPST, les étudiant-e-s ont encore moins de chances de décrocher une école vétérinaire (on passe de $30 \%$ à $13 \%$ ) mais plus de possibilités de poursuite d'études après la préparation (le taux de réussite global du concours A est de 55,8\%).

18 La population des préparationnaires est très particulière dans le paysage de l'enseignement supérieur. Les classes préparatoires accueillent majoritairement de bonne-s et très bon-ne-s élèves : la moitié d'entre eux/elles ont obtenu une mention bien ou très bien. (Lemaire, 2008). De plus, $18 \%$ des entrant-e-s en CPGE sont en avance contre $3,4 \%$ des bachelier-e-s. L'origine sociale des préparationnaires féminines et masculines 
est élevée et proche notamment en classes scientifiques (Baudelot, Dethare, Lemaire \& Rosenwald, 2003) : 44,9\% des filles et $44,6 \%$ des garçons sont originaires de milieu social supérieur ; $27,9 \%$ des filles et $28,7 \%$ des garçons de milieu intermédiaire ; $14 \%$ de filles et $15,3 \%$ de garçons de milieu populaire et enfin $13,3 \%$ de filles et $11,4 \%$ de garçons de milieu enseignant. En 2005-2006, la préparation BCPST accueille seulement 12,2\% des effectifs des élèves en classes préparatoires scientifiques (Bouhia, 2006). Tous les élèves de cette classe préparatoire possèdent un bac $S$ (99,7 \% selon Bouhia, 2006).

\section{Présentation des élèves de notre échantillon}

\section{Age des élèves}

Deux tiers des élèves sont à l'heure et un tiers a une année d'avance. Une toute petite partie a une année de retard (4,9\%). Les filles sont plus nombreuses que leurs homologues masculins à avoir un an d'avance (écart de 13 points globalement). Dans un des lycées en centre-ville on ne relève aucune différence entre les deux sexes, mais dans l'autre on constate 29 points d'écart et pour le troisième, 10 points. Ces étudiantes en avance et ayant a priori de bons dossiers scolaires, se sont-elles exclues d'un meilleur lycée en ne déposant pas leurs candidatures?

\section{Statut en classe préparatoire}

20 La majorité des élèves est interne, un peu plus les garçons (72\%) que les filles (63,7\%). Aucun-e n'est boursièr-e.

\section{Origines sociales des élèves}

21 En examinant l'ensemble des Catégories Socio-Professionnelles (CSP) des pères, nous notons que près de la moitié $(46,6 \%)$ appartient à la catégorie «Cadres et professions intellectuelles supérieures ». Les pères sont donc sur-représentés dans cette CSP qui ne compte sur le plan national que 19,1\% d'hommes en emploi (INSEE, France métropolitaine, 2009). Les pères sont aussi présents dans la CSP «professions intermédiaires » $(16,6 \%)$, toutefois en proportion moindre par rapport à la moyenne nationale (22,8 \% d'hommes en emploi). Dans les autres CSP, nous trouvons moins de $10 \%$ des pères. Nous ne constatons pas de différence significative entre les pères des filles et ceux des garçons.

Nous ne relevons pas de métiers spécifiques exercés par les pères (revoir la phrase). De nombreuses professions ont été citées mais en petit nombre. La profession d'ingénieur est celle qui est la plus nommée ( $10,6 \%$ des pères des filles et $12 \%$ des pères des garçons), puis viennent les métiers de médecin et pharmacien ( $8 \%$ des pères des filles et $18 \%$ des pères des garçons). Par contre, peu de pères exercent le métier de vétérinaire $(2,7 \%$ des pères des filles et $4 \%$ des pères des garçons). Nous ne pouvons donc pas avancer un modèle paternel pour les métiers d'ingénieur et vétérinaire.

Les mères appartiennent principalement à trois Catégories Socio-Professionnelles :

- la CSP «Cadres et professions intellectuelles supérieures » : 31,9\%, part largement supérieure à la moyenne nationale qui est de $13,8 \%$ en 2009 pour les femmes en emploi ; 
- la CSP «Professions intermédiaires » $: 27 \%$, part proche de la moyenne nationale qui est de $25,9 \%$ en 2009 ;

- CSP « Employés » : 15,3 \%, représente une part très inférieure à la moyenne nationale qui est de $47,5 \%$ en 2009. celles qui en ont au moins un ont principalement un ou des frères plus jeunes et pas encore scolarisés dans l'enseignement supérieur. Les quelques frères faisant des études supérieures suivent notamment une classe préparatoire scientifique ou une école d'ingénieurs. Nous constatons donc que les choix des élèves de notre échantillon sont proches de ceux de leurs frères poursuivant des études supérieures.

Tableau 1 : Etudes supérieures des frères

\begin{tabular}{|l|l|l|l|}
\hline \multicolumn{1}{|c|}{$\begin{array}{c}\text { Sexe } \\
\text { Etudes des frères }\end{array}$} & Garçons & Filles & Total \\
\hline Non réponse & $56 \%(28)$ & $42,5 \%(48)$ & $46,6 \%(76)$ \\
\hline Inférieur ou égal à terminale & $38 \%(19)$ & $34,5 \%(39)$ & $35,6 \%(58)$ \\
\hline Classe prépa scientifique + école ingénieurs & $14 \%(7)$ & $9,7 \%(11)$ & $11 \%(18)$ \\
\hline Fac sciences + médecine + dentaire + pharmacie & $2 \%(1)$ & $7,1 \%(8)$ & $5,5 \%(9)$ \\
\hline IUT+BTS+BTSA & $0 \%$ & $4,4 \%(5)$ & $3,1 \%(5)$ \\
\hline Fac lettres + sciences humaines+droit & $0 \%$ & $1,8 \%(2)$ & $1,2 \%(2)$ \\
\hline CAP + BEP + CAPA + BEPA & $0 \%$ & $5,3 \%(6)$ & $3,7 \%(6)$ \\
\hline Autres & $6 \%(3)$ & $5,3 \%(6)$ & $5,5 \%(9)$ \\
\hline
\end{tabular}




\begin{tabular}{|l|l|l|l|}
\hline Total & $100 \%(58)$ & $100 \%(125)$ & $100 \%(183)$ \\
\hline
\end{tabular}

Lecture : La modalité « non réponse » a été citée par 28 garçons sur 50 (total garçons), soit par $56 \%$ des garçons. Le nombre de citations est supérieur au nombre d'élèves car plusieurs réponses étaient possibles.

Note : Test du Chi2 non significatif.

Les élèves de notre échantillon sont peu nombreux à avoir au moins une sœur poursuivant des études supérieures. Parmi celles-ci, nous relevons qu'elles sont plutôt engagées dans des études universitaires dans le domaine médical et scientifique.

Tableau 2 : Etudes supérieures des sœurs

\begin{tabular}{|l|l|l|l|}
\hline \multicolumn{1}{|c|}{ Sexe } & Garçons & Filles & Total \\
\hline Etudes des sœurs & $38 \%(19)$ & $47,8 \%(54)$ & $44,8 \%(73)$ \\
\hline Inférieur ou égal à terminale & $46 \%(23)$ & $38,1 \%(43)$ & $40,5 \%(66)$ \\
\hline Classe prépa scientifique + école ingénieurs & $0 \%$ & $6,2 \%(7)$ & $4,3 \%(7)$ \\
\hline Fac sciences + médecine + dentaire + pharmacie & $12 \%(6)$ & $11,5 \%(13)$ & $11,7 \%(19)$ \\
\hline IUT + BTS + BTSA & $8 \%(4)$ & $3,5 \%(4)$ & $4,9 \%(8)$ \\
\hline Fac lettres+Sciences humaines+droit & $6 \%(3)$ & $5,3 \%(6)$ & $5,5 \%(9)$ \\
\hline \hline CAP+BEP+CAPA+BEPA & $2 \%(1)$ & $0 \%$ & $0,6 \%(1)$ \\
\hline Autres & $8 \%(4)$ & $10,6 \%(12)$ & $9,8 \%(16)$ \\
\hline Total & $100 \%(60)$ & $100 \%(139)$ & $100 \%(199)$ \\
\hline
\end{tabular}

Lecture : La modalité «non réponse » a été citée par 19 garçons sur 50 (total garçons), soit par $38 \%$ des garçons. Le nombre de citations est supérieur au nombre d'élèves car plusieurs réponses étaient possibles.

Note : Test du Chi2 non significatif.

L'examen des études scolaires des frères et sœurs met en évidence que les élèves de notre échantillon ont des parents qui connaissent bien le système scolaire et qui recherchent pour leurs enfants l'excellence scolaire par l'accès à des filières sélectives et prestigieuses comme les classes préparatoires (Gombert, 2008).

Nous constatons également que les frères sont plus investis dans les filières «classe préparatoire et école d'ingénieurs » alors que les sœurs sont plus engagées dans les études universitaires médicales. Ces choix entre frères et sœurs reflètent l'orientation générale des filles et des garçons bons élèves après un baccalauréat scientifique (Lemaire, 2005).

\section{Loisirs en classe préparatoire}

31 Dans le questionnaire, nous leur demandions à quoi était plutôt consacré leur temps libre. Nous leur proposions 4 réponses (une activité sportive, une activité liée à l'informatique, autre ou pas le temps d'avoir des loisirs) qui pouvaient être précisées (par exemple, quel type de sport). 

que c'est le cas de peu de garçons. Les filles sont-elles plus studieuses et consacrent par conséquent plus de leur temps à leurs études? Ont-elles aussi moins confiance en elles en leur réussite?

Parmi ceux et celles qui s'octroient du temps libre, on constate que certains loisirs sont significativement sexués comme le sport collectif et l'informatique qui sont plus pratiqués par les garçons. Les filles préfèrent l'équitation et dans une moindre mesure les sorties entre ami-e-s. Les autres activités sont pratiquées dans des proportions proches entre les élèves des deux sexes.

Tableau 3 : Occupation du temps libre

\begin{tabular}{|l|l|l|l|}
\hline \multicolumn{1}{|c|}{ Sexe } & Garçons & Filles & Total \\
\hline Semps libre & $30 \%(15)$ & $21,2 \%(24)$ & $23,9 \%(39)$ \\
\hline Sorties + ami-e/petit-e ami-e & $16 \%(8)$ & $25,7 \%(29)$ & $22,7 \%(37)$ \\
\hline Pas de loisirs & $6 \%(3)$ & $23 \%(26)$ & $17,8 \%(29)$ \\
\hline Equitation & $4 \%(2)$ & $20,4 \%(23)$ & $15,3 \%(25)$ \\
\hline Informatique & $24 \%(12)$ & $8 \%(9)$ & $12,9 \%(21)$ \\
\hline Musique/chant & $6 \%(3)$ & $10,6 \%(12)$ & $9,2 \%(15)$ \\
\hline Sport collectif & $18 \%(9)$ & $1,8 \%(2)$ & $6,7 \%(11)$ \\
\hline Famille & $6 \%(3)$ & $5,3 \%(6)$ & $5,5 \%(9)$ \\
\hline \hline Lecture & $6 \%(3)$ & $3,5 \%(4)$ & $4,3 \%(7)$ \\
\hline Divers & $12 \%(6)$ & $8,8 \%(10)$ & $9,8 \%(16)$ \\
\hline Total & $100 \%(60)$ & $100 \%(139)$ & $100 \%(199)$ \\
\hline
\end{tabular}

Lecture : La modalité « sport individuel » a été citée par 15 garçons sur 50 (total garçons), soit par $30 \%$ des garçons. Le nombre de citations est supérieur au nombre d'élèves car plusieurs réponses étaient possibles.

Note: Test du Chi2 =36,37 significatif.

34 Ces résultats ne sont pas surprenants puisque filles et garçons n'ont pas les mêmes pratiques culturelles. En effet, selon Octobre $(2005$, p. 3) : «En interrogeant les parents sur leurs souhaits d'activités pour leurs filles et/ou fils, on remarque qu'ils recherchent davantage pour les filles des activités sportives individuelles plutôt que collectives ou des sports « développant une approche esthétique [...] ou ayant un lien avec les animaux, comme l'équitation. [...] l'éducation au loisir est donc éminemment sexué et contribue largement à la construction sociale du genre ».

Nous pouvons d'ailleurs faire l'hypothèse qu'il existe un lien entre la pratique de l'équitation et le choix du métier vétérinaire car d'une part, la promotion 2007 de l'Ecole Nationale Vétérinaire de Toulouse compte parmi les filles, au moins $60 \%$ de cavalières alors que peu de garçons pratiquent ce sport (Sans, enquête interne ENVT, 2007). D'autre part, une enquête menée auprès de cavalières (Fontanini, 2010b) a mis en évidence que plus de la moitié de celles-ci souhaitaient devenir médecin des animaux. 


\section{Parcours d'orientation jusqu'à la classe préparatoire BCPST}

\section{Matières préférées au lycée}

Dans le questionnaire, nous leur avons demandé de citer leurs trois matières préférées au lycée.

Nous ne constatons pas de différences significatives entre les deux sexes. Nous notons néanmoins que les filles mettent plus en avant les SVT et l'écologie (écart de 11 points) et la physique/chimie (écart 7 points) que les garçons. Ces derniers préfèrent l'histoiregéographie (14 points), le français, la philosophie et une langue ancienne (écart de 8,5 points). Ces garçons montreraient une inclinaison légèrement plus prononcée pour les matières littéraires que les filles. Ce résultat ne correspond pas à la littérature sur ce sujet puisqu'en général ce sont plutôt les filles qui ont une préférence pour les matières nonscientifiques (Duru Bellat, 1990) mais cette observation correspond au niveau de l'enseignement secondaire. Les filles de notre échantillon sont dans l'enseignement supérieur et ont choisi une filière sélective scientifique ce qui peut expliquer cette différence de résultats.

Tableau 4 : Matières préférées au lycée

\begin{tabular}{|l|l|l|l|}
\hline \multicolumn{1}{|c|}{ Sexe } & Garçons & Filles & Total \\
\hline Matières préférées & $82 \%(41)$ & $93,8 \%(106)$ & $90,2 \%(147)$ \\
\hline SVT + écologie & $66 \%(33)$ & $73,5 \%(83)$ & $71,2 \%(116)$ \\
\hline Physique et/ou chimie & $50 \%(25)$ & $45,1 \%(51)$ & $46,6 \%(76)$ \\
\hline Mathématiques & $26 \%(13)$ & $26,5 \%(30)$ & $26,4 \%(43)$ \\
\hline Langue vivante & $20 \%(10)$ & $16,8 \%(19)$ & $17,8 \%(29)$ \\
\hline Sports & $26 \%(13)$ & $12,4 \%(14)$ & $16,6 \%(27)$ \\
\hline Histoire/géo/éducation civique & $20 \%(10)$ & $11,5 \%(13)$ & $14,1 \%(23)$ \\
\hline Français + philo + langues mortes & $20 \%(3)$ & $2,7 \%(3)$ & $3,7 \%(6)$ \\
\hline Autres & $100 \%(148)$ & $100 \%(319)$ & $100 \%(467)$ \\
\hline Total & & &
\end{tabular}

Lecture : La modalité « SVT+écologie » a été citée par 41 garçons sur 50 (total garçons), soit par $82 \%$ des garçons. Le nombre de citations est supérieur au nombre d'élèves car plusieurs réponses étaient possibles.

Note: Test du Chi2 non significatif.

\section{Choix de la terminale scientifique}

La plupart des raisons évoquées par les élèves des deux sexes de notre échantillon sont très proches pour leur choix d'une terminale scientifique. Nous relevons toutefois que les filles citent plus que les garçons leur désir de suivre des études scientifiques post- 
baccalauréat (écart de 20 points) et ces derniers avancent davantage les nombreux débouchés d'un baccalauréat scientifique.

Selon Lemaire (2005), les filles sont plus nombreuses que les garçons à savoir ce qu'elles veulent faire comme métier plus tard. Nous pouvons supposer qu'il en est de même pour les filles de notre échantillon et qu'elles avaient donc davantage déjà anticipé un métier scientifique comparativement aux garçons.

Tableau 5 : Raisons pour le choix de la terminale

\begin{tabular}{|l|l|l|l|}
\hline \multicolumn{1}{|c|}{$\begin{array}{l}\text { Sexe } \\
\text { Choix terminale S }\end{array}$} & Garçons & Filles & Total \\
\hline Désir suivre études scientifiques après Bac & $58 \%(29)$ & $78,8 \%(89)$ & $72,4 \%(118)$ \\
\hline \hline Attrait SVT & $52 \%(26)$ & $57,5 \%(65)$ & $55,8 \%(91)$ \\
\hline Pas attiré-e par disciplines littéraires et commerciales & $44 \%(22)$ & $38,1 \%(43)$ & $39,9 \%(65)$ \\
\hline Bac S = plus de débouchés & $46 \%(23)$ & $33,6 \%(38)$ & $37,4 \%(64)$ \\
\hline Attrait pour physique-chimie & $26 \%(13)$ & $24,8 \%(28)$ & $25,2 \%(41)$ \\
\hline Attrait pour les maths & $18 \%(9)$ & $20,4 \%(23)$ & $19,6 \%(32)$ \\
\hline \hline Pas assez bon-ne pour une autre filière & $6 \%(3)$ & $2,7 \%(3)$ & $3,7 \%(6)$ \\
\hline Autres & $8 \%(4)$ & $9,7 \%(11)$ & $9,2 \%(15)$ \\
\hline Total & $100 \%(123)$ & $100 \%(300)$ & $100 \%(429)$ \\
\hline
\end{tabular}

Lecture : La modalité « attrait SVT » a été citée par 26 garçons sur 50 (total garçons), soit par 52 \% des garçons. Le nombre de citations est supérieur au nombre d'élèves car plusieurs réponses étaient possibles.

Note : Test du Chi2 non significatif.

\section{Obtention du Baccalauréat}

La quasi-totalité des élèves $(98,8 \%)$ a obtenu leur baccalauréat l'année précédant leur entrée en préparation.

41 Deux élèves sur cinq ( $39,8 \%$ de filles et $42 \%$ de garçons) avaient opté pour les SVT comme spécialité au baccalauréat. Le choix des mathématiques $(26,5 \%$ de filles et $28 \%$ de garçons), de la physique-chimie ( $28,3 \%$ de filles et $28 \%$ de garçons) et de la biologieécologie ( $5,3 \%$ de filles et $2 \%$ de garçons) a été fait dans les mêmes proportions et entre les deux sexes.

42 Notre échantillon est représentatif de la distribution des élèves sur le plan national selon leur spécialité au baccalauréat puisqu'en 2005, 30,2 \% d'élèves de première année en préparation BCPST avaient suivi la spécialité « mathématiques », $38 \%$ « Sciences de la Vie et de la Terre » et 27,9\% « physique-chimie » (Bouhia, 2005).

43 Nous ne constatons pas de différence significative entre les deux sexes pour cet indicateur. 
La majorité des préparationnaires est arrivée en CPGE bien dotée scolairement puisque près de huit élèves sur dix (78 \%) sont détenteurs d'une mention «Bien » ou " Très bien » avec peu de différences (non significatif) entre les filles (78,7 \%) et les garçons (76 \%).

En comparant avec la répartition des bachelier-e-s de la session 2005 entrants en première année de classes préparatoires BCPST selon la mention au baccalauréat (Bouhia, 2005), nous notons que la part des élèves sur le plan national s'élève à $18 \%$ pour la mention «Très bien » et à $38 \%$ pour la mention «Bien ». Les préparationnaires BCPST des 3 lycées toulousains étudiés sont donc mieux dotés scolairement que ceux et celles en France métropolitaine + DOM (Public + Privé).

\section{Choix de la classe préparatoire BCPST}

La question proposée aux élèves était la suivante : « Comment s'est fait votre choix d'aller en classe préparatoire BCPST ? ». Ils/elles pouvaient cocher 3 réponses fermées maximum sur 9 proposées, sans ordre de préférence.

Tableau 6 : Choix de la classe préparatoire

\begin{tabular}{|l|l|l|l|}
\hline \multicolumn{1}{|c|}{$\begin{array}{c}\text { Sexe } \\
\text { Choix classe préparatoire }\end{array}$} & Garçons & Filles & Total \\
\hline Désir intégration école vétérinaire & $52 \%(26)$ & $72,6 \%(82)$ & $66,3 \%(108)$ \\
\hline Pas souhait d'aller à l'université & $36 \%(18)$ & $31 \%(35)$ & $32,5 \%(53)$ \\
\hline Désir intégration école agronomie & $48 \%(24)$ & $21,2 \%(24)$ & $29,4 \%(48)$ \\
\hline Voie royale & $18 \%(9)$ & $23,9 \%(27)$ & $22,1 \%(36)$ \\
\hline Personnes entourage amical et familial & $4 \%(2)$ & $8 \%(9)$ & $6,7 \%(11)$ \\
\hline Conseils profs + COP & $12 \%(6)$ & $5,3 \%(6)$ & $7,4 \%(12)$ \\
\hline Autres & $20 \%(10)$ & $7,1 \%(8)$ & $11 \%(18)$ \\
\hline Total & $100 \%(95)$ & $100 \%(191)$ & $100 \%(286)$ \\
\hline
\end{tabular}

Lecture : La modalité « désir école vétérinaire » a été citée par 26 garçons sur 50 (total garçons), soit par $52 \%$ des garçons. Le nombre de citations est supérieur au nombre d'élèves car plusieurs réponses étaient possibles. Note: Test du Chi2 $=17,97$ significatif.

47 La moitié des garçons désire intégrer une école vétérinaire et une autre moitié, une école d'agronomie alors que les filles sont plus tentées par une école vétérinaire (7 sur 10) et seulement une sur cinq désire intégrer une école d'agronomie. Nous relevons ainsi une plus forte motivation pour les écoles vétérinaires de la part des filles que des garçons.

Nous notons que les élèves des deux sexes avancent à parts égales les autres motifs liés à leurs choix, notamment leur non désir d'entrer à l'université et la faible influence de leur entourage (familiale et au lycée) 


\section{Candidatures dans quels lycées?} dans d'autres lycées :

- un manque de confiance en elles et une peur de ne pas être acceptées, donc elles multiplient leurs chances au maximum ;

- une volonté farouche de suivre une préparation BCPST pour poursuivre des études précises après comme celles de vétérinaire ;

- les filles sont plus mobiles et autonomes que les garçons, elles auraient moins peur de quitter leur domicile familial.

\section{Projets professionnels}

\section{Quelle école intégrer après la classe préparatoire ?}

Les élèves ont tous et toutes une idée au bout d'un mois des établissements convoités après leur préparation. Les garçons sont quasiment autant attirés par les écoles d'agronomie et les Ecoles Nationales Vétérinaires (ENV), à égalité ensuite par les Ecoles Nationales Supérieures (ENS) et les Ecoles Nationales d'Ingénieurs des Travaux Agricoles (ENITA), enfin par diverses autres écoles. Huit filles sur dix espèrent poursuivre leurs études dans une ENV. Elles sont moins tentées par une école d'agronomie qu'une école vétérinaire (écart de 20 points). Les ENS, ENITA et diverses autres écoles sont moins convoitées par les élèves féminines.

Les filles apparaissent comme polarisées en premier sur les écoles vétérinaires et en second sur les écoles d'agronomie alors que les garçons ont des souhaits plus diversifiés.

Tableau 8 : Quelle école après la préparation ?

\begin{tabular}{|l|l|l|l|}
\hline \multicolumn{1}{|c|}{ Sexe } & Garçons & Filles & Total \\
\hline Ecoles & & & \\
\hline Ecoles agronomie & $70 \%(35)$ & $61,1 \%(69)$ & $63,8(104)$ \\
\hline \hline Ecoles Normales Supérieures & $38 \%(19)$ & $33,6 \%(38)$ & $35 \%(57)$ \\
\hline ENITA & $40 \%(20)$ & $22,1 \%(25)$ & $27,6 \%(45)$ \\
\hline Autres & $24 \%(12)$ & $14,2 \%(16)$ & $17,2 \%(28)$ \\
\hline
\end{tabular}




\begin{tabular}{|l|l|l|l|}
\hline Total & $100 \%(118)$ & $100 \%(240)$ & $100 \%(358)$ \\
\hline
\end{tabular}

Lecture : La modalité « Ecoles Nationales Vétérinaires » a été citée par 32 garçons sur 50 (total garçons), soit par $64 \%$ des garçons. Le nombre de citations est supérieur au nombre d'élèves car plusieurs réponses étaient possibles.

Note : Test du Chi2, non significatif.

\section{Profession souhaitée?}

Nous leur demandions de nous dire quelle profession les tente le plus. Une seule réponse était possible parmi les 4 proposées : ingénieur-e, vétérinaire, enseignant-e, autre.

Tableau 9 : Profession souhaitée

\begin{tabular}{|l|l|l|l|}
\hline \multicolumn{1}{|c|}{ Sexe } & Garçons & Filles & Total \\
\hline Ingofession & & & \\
\hline Vétérinaire & $50 \%(25)$ & $69 \%(78)$ & $63,2 \%(103)$ \\
\hline Enseignant-e & $4 \%(2)$ & $0,9 \%(1)$ & $1,8 \%(3)$ \\
\hline Autres & $14 \%(7)$ & $13,3 \%(15)$ & $13,5 \%(22)$ \\
\hline Total & $100 \%(70)$ & $100 \%(141)$ & $100 \%(211)$ \\
\hline
\end{tabular}

Lecture : La modalité « Ingénieur agronome » a été citée par 36 garçons sur 50 (total garçons), soit par $72 \%$ des garçons. Le nombre de citations est supérieur au nombre d'élèves car plusieurs réponses étaient possibles.

Note: Test du Chi2 =9,11 très significatif.

En moyenne, les élèves ont donné un peu plus d'une réponse par personne (1,4 réponses pour les garçons et 1,2 pour les filles). Nous pouvons penser qu'ils/elles n'ont pas encore fait définitivement leur choix et surtout qu'ils/elles ne veulent pas se polariser sur un seul métier en cas d'échec au concours. Nous retrouvons toutefois toujours la même tendance à savoir que les garçons sont plus attirés par le métier d'ingénieur agronome (écart de 22 points) que vétérinaire et les filles par ce dernier (écart de 19 points).

57 Nous avons également relevé cette préférence des filles en classes préparatoires TB (Technologie-Biologie) et ATS Biologie (Années préparatoires Spéciales pour Technicien Supérieur) pour les écoles vétérinaires (Fontanini, 2010a).

Ces choix professionnels précis notamment de la part des filles semblent propres à ce type de préparation car comme le souligne Limann $(2009$, p. 55) à propos des classes préparatoires mathématiques et physique : « Les bacheliers s'engagent rarement en prépa dans la perspective d'exercer un métier particulier. Rares sont ceux qui veulent consciemment devenir ingénieur (...) ».

\section{Sur quels critères choisir sa future profession?}

Les élèves étaient invité-e-s à répondre à cette question « Comment s'est fait ou se fera le choix de votre future profession? ». Ils/elles devaient choisir 2 réponses maximum parmi 9 réponses fermées proposées. 
Tableau 10 : Critères de choix

\begin{tabular}{|l|l|l|l|}
\hline \multicolumn{1}{|c|}{$\begin{array}{c}\text { Sexe } \\
\text { Critères de choix }\end{array}$} & Garçons & Filles & Total \\
\hline Par goût & $76 \%(38)$ & $83,2 \%(94)$ & $\mathbf{8 1} \%(\mathbf{1 3 2})$ \\
\hline Par vocation & $34 \%(17)$ & $47,8 \%(54)$ & $\mathbf{4 3 , 6} \%(\mathbf{7 1})$ \\
\hline Pour ses avantages financiers & $20 \%(10)$ & $6,2 \%(7)$ & $\mathbf{1 0 , 4} \%(\mathbf{1 7})$ \\
\hline Pour une conciliation famille/travail & $12 \%(6)$ & $8 \%(9)$ & $\mathbf{9 , 2} \%(\mathbf{1 5})$ \\
\hline Pour avoir du temps libre pour loisirs & $8 \%(4)$ & $5,3 \%(6)$ & $\mathbf{6 , 1} \%(\mathbf{1 0})$ \\
\hline Pour une installation en libéral & $10 \%(5)$ & $3,5 \%(4)$ & $5,5 \%(9)$ \\
\hline On trouve du travail dans cette profession & $4 \%(2)$ & $4,4 \%(5)$ & $\mathbf{4 , 3 \% ( 7 )}$ \\
\hline \hline autres & $6 \%(3)$ & $5,3 \%(6)$ & $5,5 \%(9)$ \\
\hline Total & $\mathbf{1 0 0 \% ( 8 5 )}$ & $\mathbf{1 0 0 \% ( 1 8 5 )}$ & $\mathbf{1 0 0 \% ( 2 7 0 )}$ \\
\hline
\end{tabular}

Lecture : La modalité «par goût » a été citée par 38 garçons sur 50 (total garçons), soit par 76 \% des garçons. Le nombre de citations est supérieur au nombre d'élèves car plusieurs réponses étaient possibles.

Note: Test du Chi2 $=11,51$ peu significatif.

Le "goût" pour une profession est la première motivation avancée par la grande majorité des élèves des deux sexes. Presque la moitié des filles évoque en second la vocation contre un tiers de garçons (écart de 14 points); ces derniers mettant plus en avant les avantages financiers (écart de 14 points). Les autres raisons sont avancées dans des proportions proches par les élèves des deux sexes.

61 Nous retrouvons, comme dans de nombreuses études, la moindre propension des filles à choisir une profession pour ses avantages financiers contrairement aux garçons. Le modèle économique $\mathrm{du}$ "breadwinner» (l'homme, principal pourvoyeur de ressources), avancé par Parsons dans les années 1950, persiste malgré une percée des femmes dans les études supérieures et sur le marché du travail depuis plus de trente ans. Les filles continuent, en effet, à plus choisir une profession pour son intérêt (goût, vocation) que pour les revenus qu'elle procure (Veleine, 2004). Cette différence entre les deux sexes se construit au cours de l'éducation et de la socialisation. Il est encore attendu communément que les femmes s'occupent davantage des enfants et que les hommes s'investissent professionnellement.

\section{Conclusion}

Le profil social des filles et des garçons en classe préparatoire BCPST est proche sur de nombreux aspects. Nous relevons des origines sociales élevées, des frères et sœurs investi-e-s dans des études supérieures sélectives (plutôt les classes préparatoires pour les frères et le secteur médical pour les sœurs). Ces préparationnaires ont peu de modèles proches dans leurs entourages des professions de vétérinaires et d'ingénieurs. Le profil scolaire est sensiblement équivalent. Nous notons juste une prépondérance des filles en 
avance. Ainsi, cette minorité de garçons a des caractéristiques proches de cette majorité de filles.

63 Nous relevons toutefois des différences entre ces élèves des deux sexes concernant notamment les projets professionnels. Les garçons se dirigent davantage vers cette préparation car c'est une voie d'excellence alors que les filles choisissent plus ces études en ayant une idée professionnelle précise, notamment le métier de vétérinaire.

Il existe probablement d'autres raisons explicatives. Nous pouvons avancer le fait que les filles aiment plus la biologie que les garçons (Veleine, 2004) ce qui les pousse davantage vers cette filière d'études supérieure prestigieuse. D'autre part, les parents laissent probablement plus les filles excellentes que les garçons excellents en terminale scientifique se diriger vers une préparation BCPST ; les garçons étant davantage guidés vers une classe préparatoire de mathématiques et physique (Ferrand, Imbert \& Marry, 1996).

Pour compléter cette étude, nous avons pour projet d'enquêter auprès d'élèves de terminales scientifiques pour connaitre les raisons ou leur rejet de projet vers cette préparation. Ainsi, nous espérons comprendre encore mieux pourquoi les garçons sont de moins en moins nombreux et les filles de plus en plus présentes dans cette filière d'études supérieures.

\section{BIBLIOGRAPHIE}

Adangnikou, N., \& Paul, J.-J. (2004). Cela vaut-il la peine de faire une prépa ? Une réponse à partir de l'insertion des ingénieurs. Net.Doc.7, 1-32.

Baudelot, C., \& Establet, R. (1992). Allez les filles. Paris : Seuil.

Baudelot C., Dethare, B., Lemaire, S., \& Rosenwald, F. (2003). Les CPGE au fil du temps.

Communication au colloque Démocratie, classes préparatoires et grandes écoles. ENS, 16 et 17 mai.

Belhoste, B. (2003). Une histoire des CPGE. Communication au colloque Démocratie, classes préparatoires et grandes écoles. ENS, 16 et 17 mai.

Boudon, R. (1973). L'inégalité des chances. La mobilité sociale dans les sociétés industrielles. Paris : Armand Colin.

Bouhia, R. (2006). Les étudiants en classes préparatoires aux grandes écoles. Note d'information du MESNR, 06-23, 6 pages.

Bourdieu, P. (1970). La Reproduction. Paris : Minuit.

Bourdieu, P. (1981). Le sens pratique, Paris : Minuit.

Bourdieu, P. (1989). Noblesse d'Etat et esprit de corps. Paris : Minuit.

Boutinet, J.-P. (2001). Anthropologie du projet. Paris : PUF. 
Buisson-Fenet, H., \& Landrier, S. (2008). En être ou pas ? Discrimination positive et révélation du rapport au savoir. Le cas d'une « prépa ZEP » de province. Education et Sociétés, 21(1), 67-80.

Dardelet, C. (2010). Ouverture sociale des grandes écoles - Livre blanc des pratiques, Premiers résultats et perspectives. Paris : La Documentation française.

Delavault, H. (1998). La place des filles dans une filière de formation des cadres. Les grandes écoles scientifiques. Rapport Association Française des Femmes \& Demain la Parité.

Duru Bellat, M. (1990). L'école des filles. Quelle formation pour quels rôles sociaux ? Paris : L'Harmattan.

Duru Bellat, M. (1994). Filles et garçons à l'école, approches sociologiques et psychosociales. Revue Française de Pédagogie, 109, 111-141.

Duru Bellat, M. (1995). Filles et garçons à l'école, approches sociologiques et psychosociales. Revue Française de Pédagogie, 110, 75-109.

Dutercq, Y. (2008). Former des élites dans un monde incertain. Education et Sociétés (coord), $\mathrm{n}^{\circ} 1$.

Ferrand, M., Imbert F., \& Marry, C. (1996), Femmes et sciences : une équation improbable? L'exemple des normaliennes scientifiques et des polytechniciennes. Formation Emploi, 55, 3-18.

Fontanini, C. (1999). Les filles face aux classes de mathématiques supérieures et spéciales : Analyse des déterminants des choix d'une filière considérée comme atypique à leur sexe. Thèse de doctorat Sciences de l'Education, Université de Bourgogne.

Fontanini, C. (2008). Jeux vidéo et jouets : un lien avec la féminisation du métier vétérinaire ? Les Cahiers du CERFEE, 25, 147-165.

Fontanini, C. (2009). La socialisation des filles vers le monde des animaux. Communication au congrès de la Société Française de Psychologie Toulouse "Psychologie et enjeux de société », Université Toulouse le Mirail, 17-19 juin.

Fontanini, C. (2010a). Les classes préparatoires scientifiques TB et ATS : des prépas mal connues et reconnues. Les Cahiers du CERFEE, 29, 59-72.

Fontanini, C. (2010b). Presse et livres de jeunesse pour fillettes et adolescentes, pratique de l'équitation : un lien avec la féminisation du métier vétérinaire ? In V. Rouyer, S. Croity-Belz \& Y. Preteur(Eds.), Socialisation de genre (pp. 73-84). Toulouse : Eres.

François-Poncet, C.-M., \& Braconnier, A. (1998). Classes préparatoires. Des étudiants pas comme les autres. Paris : Bayard.

Gauthier, G. (2008). Orientation et insertion professionnelle: vers un rééquilibrage entre femmes et hommes dans tous les métiers. Rapport d'activité du Sénat $n^{\circ} 404$. Annexe au procès-verbal de la séance du 18 juin.

Gombert, P. (2008). L'école et ses stratèges. Les pratiques éducatives des nouvelles classes supérieures. Rennes : PUR.

Guichard, J. (1993). L'école et les représentations d'avenir des adolescents. Paris : PUF.

Huerre, P. (2005). Faut-il plaindre les bons élèves? Le prix de l'excellence. Paris : Hachette.

Jacques, M.-H. (2003). Garçons et filles de classes terminales : le filtre sexué des représentations du cursus et des intentions d'orientation post-baccalauréat. Carrefours de l'éducation, 15, 62-81.

Lemaire, S. (2005). Les premiers bacheliers du panel : aspirations, image de soi et choix d'orientation. Education et formation, 72, 137-153. 
Lemaire, S. (2008). Disparités d'accès et parcours en classes préparatoires. Note d'information MESR, 08-16, 6 pages.

Limann, T. (2009). Classé X. Petits secrets des classes prépas. Paris : La Découverte.

Mosconi, N. (1994). Femmes et savoir : La société, l'école et la division sexuelle des savoirs. Paris : L'Harmattan.

Mosconi, N., \& Stevanovic, B. (2007). Genre et avenir. Les représentations des métiers chez les adolescentes et les adolescents. Paris : L'Harmattan.

Octobre, S. (2005). La fabrique sexuée des goûts culturels. Construire son identité de fille ou de garçon à travers les activités culturelles. Développement culturel, 150, 1-10.

Pauly, E., \& Mandry, P. (2003). Bien choisir sa classe préparatoire. $\mathrm{n}^{\circ}$ 202, série : études, L'Etudiant.

Pons, A. (2007). Les étudiants en classe préparatoire aux grandes écoles. Note d'information MESR, 07-37.

Rapport sur les concours A, filière BCPST (2005). www.concours-agro-veto.net.

Ministère de l'Éducation Nationale et Ministère de l'Enseignement Supérieur et de la Recherche (2010). Repères et références statistiques sur les enseignements, la formation et la recherche.

Sans, P., \& Darré, R. (2007). Les représentations et motivations des étudiants et des étudiantes en $1^{1}$ ère année d'école d'ingénieur-e ou vétérinaire. Un $=U n e$, Lettre du réseau "Egalité des chances entre les femmes et les hommes dans l'enseignement supérieur agricole », 6, 8 pages.

Stevanovic, B. (2006). La mixité dans les écoles d'ingénieurs. Paris : l'Harmattan.

Tourre-Malen, C. Femmes à cheval. Paris : Belin.

Veleine, C. (2004). L'égalité des chances entre les filles et les garçons : la première insertion professionnelle des ingénieurs agronomes. Rapport de la DGER, Ministère de l'agriculture, de l'alimentation, de la pêche et de la ruralité, décembre.

Vouillot, F. (2007). L'orientation aux prises avec le genre. Travail, genre et sociétés,18, 87-108.

\section{RÉSUMÉS}

Cet article a pour objectif de comprendre pourquoi les filles sont nombreuses (70\%) à s'engager vers la classe préparatoire scientifique Biologie, Chimie, Physique, Sciences de la Terre (BCPST) alors qu'elles restent minoritaires dans les autres préparations scientifiques (Pons, 2007). Comment se sont construits pour ces étudiants et étudiantes, leur projet et leur parcours scolaire vers cette classe préparatoire "bio-véto"? Quelles sont leurs motivations pour cette filière menant aux professions de vétérinaire et d'ingénieur agronome? Quelles sont les écoles les plus convoitées? Quels sont leurs projets professionnels? Sont-ils différents entre les filles et les garçons? Pour tenter de répondre à ces questions, nous avons mené une recherche par questionnaire, courant septembre 2008, auprès de 163 élèves de 4 classes préparatoires BCPST de première année (113 filles et 50 garçons) des 3 lycées toulousains proposant cette filière dans l'académie. Les filles sont largement majoritaires en classes préparatoires BCPST car leurs débouchés apparaissent comme plus attractifs pour les filles, notamment les écoles vétérinaires.

This paper examines why girls are more numerous (70\%) in the scientific preparatory classes BCPST (Biology, Chemistry, Physics and Earth) whereas they are a minority in the other scientific preparatory classes (Pons, 2007). How have female and male built their occupational preferences 
and their school choices for this preparatory class "biology - vet science"? What are their motivations for this pathway which prepares for veterinarian and agricultural engineer? What are the most desired schools? What are their professional plans? Are they different between girls and boys? In order to answer these questions, a questionnaire survey was conducted in September 2008, on a sample of 163 pupils (113 girls and 50 boys) from 4 first year preparatory classes BCPST in 3 Toulousian high schools proposing this school pathway in the academy (regional education authority). The girls are widely majority in preparatory classes BCPST because their career pathways appear more attractive for the girls, particularly the veterinarian schools.

INDEX

Keywords : gender, university

Mots-clés : enseignement supérieur, genre, orientation

\section{AUTEUR}

\section{CHRISTINE FONTANINI}

Maîtresse de conférences - Laboratoire LIRDEF (EA4739) - Université Paul Valéry, Montpellier, France. 